ARTICLE

https://doi.org/10.1038/s41467-019-11244-3

\title{
Brainstem and spinal cord MRI identifies altered sensorimotor pathways post-stroke
}

Haleh Karbasforoushan (10 1,2, Julien Cohen-Adad (1) ${ }^{3,4}$ \& Julius P.A. Dewald ${ }^{1,2,5}$

Damage to the corticospinal tract is widely studied following unilateral subcortical stroke, whereas less is known about changes to other sensorimotor pathways. This may be due to the fact that many studies investigated morphological changes in the brain, where the majority of descending and ascending brain pathways are overlapping, and did not investigate the brainstem where they separate. Moreover, these pathways continue passing through separate regions in the spinal cord. Here, using a high-resolution structural MRI of both the brainstem and the cervical spinal cord, we were able to identify a number of microstructurally altered pathways, in addition to the corticospinal tract, post stroke. Moreover, decreases in ipsi-lesional corticospinal tract integrity and increases in contra-lesional medial reticulospinal tract integrity were correlated with motor impairment severity in individuals with stroke.

\footnotetext{
${ }^{1}$ Interdepartmental Neuroscience Program, Feinberg School of Medicine, Northwestern University, Chicago, 60611 IL, USA. ${ }^{2}$ Department of Physical Therapy and Human Movement Sciences, Feinberg School of Medicine, Northwestern University, Chicago, 60611 IL, USA. ${ }^{3}$ NeuroPoly Lab, Institute of Biomedical Engineering, Polytechnique Montréal, Montréal, H3T $1 \mathrm{~J} 4$ QC, Canada. ${ }^{4}$ Functional Neuroimaging Unit, CRIUGM, University of Montreal, Montreal, H3T 1J4 QC, Canada. ${ }^{5}$ Department of Biomedical Engineering, McCormick School of Engineering, Northwestern University, Evanston, 60208 IL, USA. Correspondence and requests for materials should be addressed to H.K. (email: Haleh@u.northwestern.edu) or to J.P.A.D. (email: j-dewald@northwestern.edu)
} 
S ubcortical unilateral strokes affecting the internal capsule or basal ganglia are the most common of all strokes and usually result in hemiparesis of the contralateral arm and leg. Previous human brain imaging studies of unilateral subcortical stroke have frequently reported damage to the corticospinal tract ${ }^{1-4}$. Cross-sectional studies also reported this damage to be associated with greater motor deficits as well as worse motor recovery in these individuals ${ }^{5-8}$. However, less is known about other altered sensorimotor pathways post unilateral stroke and their role in motor impairments. An important reason for this lack of knowledge is that previous studies have only investigated the morphological changes in the brain, where the majority of descending and ascending brain pathways (e.g., corticospinal tract, cortico-bulbospinal tracts, dorsal column medial lemniscus) mostly overlap and are not distinguishable with currently available imaging techniques. Sensorimotor pathways, in fact, delineate from each other in the brainstem ${ }^{9-12}$. Moreover, these tracts continue travelling through separate regions in the spinal $\operatorname{cord}^{9,10}$.

It is also noteworthy to mention that numerous functional neuroimaging studies of stroke have reported increased neural activity in motor cortices of both lesioned and non-lesioned hemisphere during hand/arm movement ${ }^{13-16}$. They have also reported that the more severe the impairment, the greater the activity in the contralesional hemisphere compared to the ipsilesional (i.e., a shift of activity to non-lesioned hemisphere) ${ }^{17-23}$. However, it is still unclear what descending motor pathway allows the contralesional motor cortex to control the ipsilateral paretic arm, although one proposed idea from human and animal studies suggests ${ }^{24-28}$ brainstem ipsilaterally projecting motor pathways (i.e., reticulospinal tracts or vestibulospinal tracts) may play this role.

Based on magnetic resonance imaging (MRI), diffusion tensor imaging (DTI) can probe white matter microstructure by providing information on the direction and degree of water diffusivity in white matter tracts ${ }^{29}$. The degree of anisotropy (fractional anisotropy: FA) of water in the white matter tracts reflects their level of integrity ${ }^{30,31}$. Evidence from human brain imaging studies suggests that white matter integrity could change with experience. For example, damage or degeneration to a tract is shown to be associated with a decrease in its white matter integrity $(\mathrm{FA})^{1-3,32-34}$. Likewise, training-induced or experiencedependent increases in white matter integrity have also been reported in humans ${ }^{35-37}$. Moreover, recent developments in MRI of the spinal cord have opened new doors to further studies of the human nervous system ${ }^{38-40}$. High-resolution DTI of cervical spinal cord and novel analyses approaches provide means of investigating the spinal cord white matter tracts. DTI's ability to determine long-term changes in white matter structure and its recent developments in the cervical spinal cord ${ }^{38-40}$ makes this an excellent method for investigating altered sensorimotor pathways in the brainstem and spinal cord in individuals with chronic stroke.

To this end, we used high-resolution structural MRI of the brainstem and cervical spinal cord and unbiased voxel-wise analyses to identify all altered sensorimotor pathways post unilateral subcortical stroke. Subsequently, the link between microstructural changes in pathways and paretic upper limb motor impairments (Fugl-Meyer Assessment (FMA)) was examined in the stroke participants. It was hypothesized that individuals with stroke would show reduced white matter integrity in a number of sensorimotor pathways travelling through subcortical regions of lesioned hemisphere, in addition to the corticospinal tract. Furthermore, an increased white matter integrity in some brainstem ipsilaterally projecting pathways from the non-lesioned hemisphere was expected. Our results indicate a significant decrease of white matter integrity in corticospinal tract, lateral and medial reticulospinal tracts, descending medial longitudinal fasciculus, tectospinal tract, and cuneate and gracile fasciculi related to the lesioned hemisphere. Furthermore, the results from brainstem and cervical spinal cord DTI analyses indicate a significant increase in the white matter integrity of medial reticulospinal tract at the side of contralesional hemisphere, which projects ipsilaterally to the paretic (contralesional) limbs. The decreased white matter integrity of ipsilesional corticospinal tract and increased white matter integrity of contralesional medial reticulospinal tract are correlated with upper limb impairment severity in individuals with stroke.

\section{Results}

Demographics. Of the subjects recruited, five stroke participants and one healthy control were excluded from the study due to brainstem, bilateral or cortical lesions, or poor-quality image. Therefore, the final sample consisted of 31 individuals with stroke and 31 healthy controls. Demographics of two groups are presented in Table 1. The stroke group had more African-American participants than the controls group $\left(x^{2}=8.72, p=0.01\right)$. The two groups were well matched on age and gender. The lesion map of individuals with stroke is presented in Supplementary Fig. 1.

Brainstem white matter integrity in stroke. Tracts with significant white matter integrity changes in the brainstem of individuals with stroke compared to controls are presented in Fig. 1 (threshold-free cluster enhancement $p=0.05$ ). In the brainstem

\begin{tabular}{|c|c|c|c|c|c|c|c|}
\hline$N$ & 31 & & 31 & & & & \\
\hline $\begin{array}{l}\text { Ethnicity (White: } \\
\text { AA:Other) }\end{array}$ & $26: 4: 1$ & & 15:13:3 & & 8.72 & 4 & 0.012 \\
\hline $\begin{array}{l}\text { Affected side of } \\
\text { body (left:right) }\end{array}$ & Mean & SD & Mean & SD & $t$ & df & $p$ Value \\
\hline Age & 61.61 & 9.45 & 59.83 & 8.97 & 0.76 & 60 & 0.452 \\
\hline Years since onset & - & - & 11.48 & 7.65 & - & 30 & - \\
\hline Fugl-Meyer ${ }^{a}$ & - & - & 31.00 & 15.78 & - & 29 & - \\
\hline
\end{tabular}


a

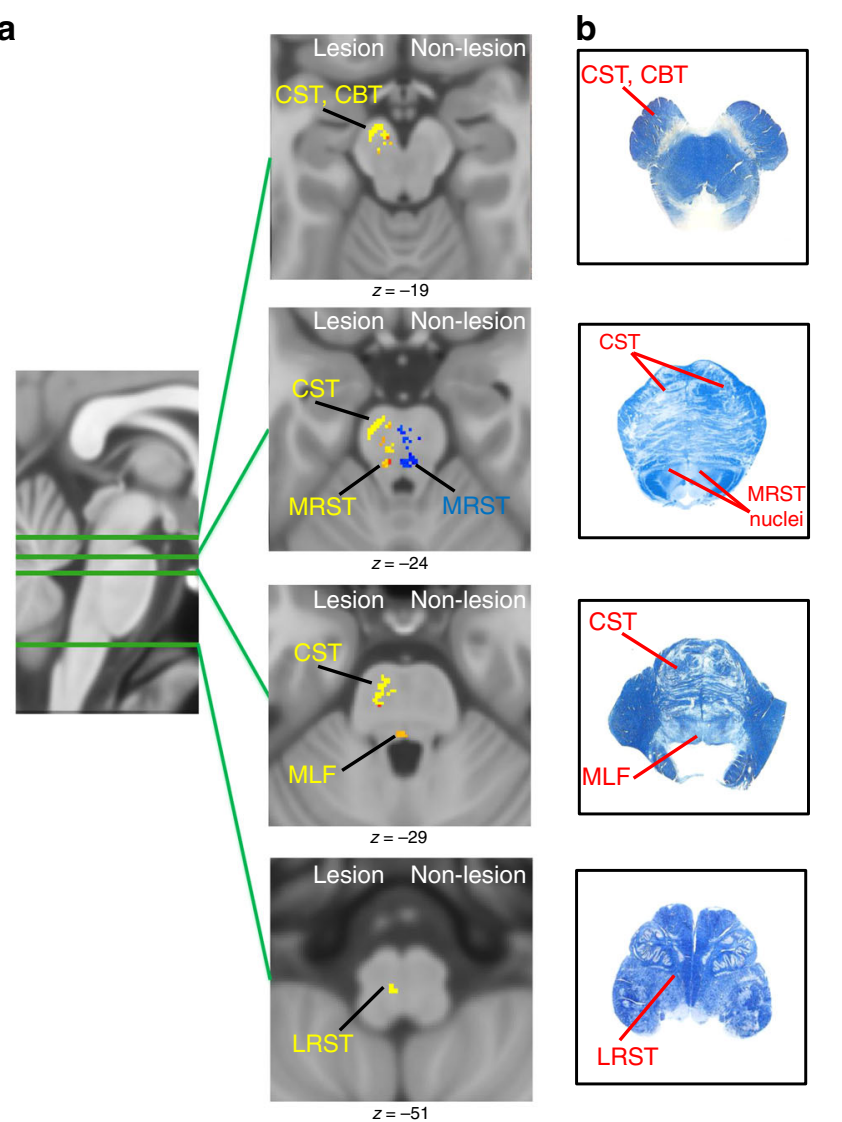

C
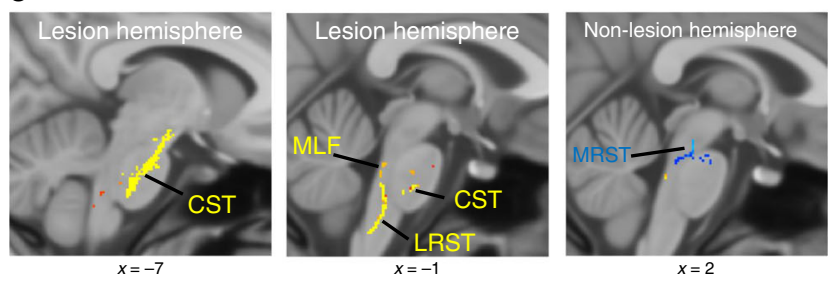

Stroke $<$ Control 0.001 0.05 105 $p$ the non-lesioned hemisphere, an increased white matter integrity in individuals with stroke was found in the medial reticulospinal tract, which receives projections from non-lesioned motor cortices (Fig. 1, tracts in blue-light blue color). The locations of sensorimotor tracts in the brainstem were determined with the help of Gray's anatomy ${ }^{9}$, Haines' neuroanatomy ${ }^{10}$, and Paxinos' brainstem anatomy ${ }^{11,41}$. Fig. $1 \mathrm{~b}$ shows the location of tracts on the brainstem atlas, focusing on the sensorimotor pathways under investigation, for simplicity.

We then tested for any associations between these white matter alterations and clinical motor assessments in the paretic upper limb (Fugl-Meyer Motor Assessment score). Figure 2 illustrates the tracts with significant correlation between white matter integrity changes and motor impairment in individuals with stroke. Decreases in corticospinal tract integrity at the lesioned hemisphere and increases in medial reticulospinal tract integrity at the non-lesioned hemisphere in individuals with stroke were shown to be significantly correlated with their motor impairment severity (Fig. 2).

Spinal cord white matter integrity in stroke. Figure 1 shows the tracts with significant white matter integrity changes in cervical spinal cord in individuals with stroke compared to the controls. The results from the cervical spinal cord analysis indicated a significant decrease in white matter integrity of lateral corticospinal tract, tectospinal tract, and cuneate and gracile fasciculi at the paretic (contralesional) side in individuals with stroke. The stroke participants also demonstrated a significantly decreased white matter integrity in a few smaller clusters, which, to our current knowledge of spinal cord pathways locations, appear to belong to the medial reticulospinal tract, medial longitudinal fasciculus, and medial corticospinal tract at the non-paretic side of body. Of note, these pathways project ipsilaterally from the lesioned hemisphere (Fig. 3a, in red-yellow color). Consistent with results from the brainstem analyses, patients showed a significant increase in white matter integrity in a small cluster that, to our current knowledge of spinal cord pathways, belongs to the medial reticulospinal tract on paretic (contralesional) side, which projects ipsilaterally from the non-lesioned hemisphere (Fig. 3b, in blue-light blue color). All resulted tracts are thresholded with a voxel-wise $p$ value of 0.005 and corrected for cluster size with a $p$ value of 0.05 . Figure $3 \mathrm{c}$ illustrates the high-resolution spinal cord white matter atlas derived from the Gray's anatomy atlas, adapted from ref. ${ }^{42}$

Testing for any associations between these white matter differences in cervical spinal cord and clinical assessments of motor impairment in individuals with stroke, we found that the decreased white matter integrity of lateral corticospinal tract and increased white matter integrity of medial reticulospinal tract were correlated with motor impairment severity in these individuals (Fig. 3d). In other words, a greater severity of motor impairment was correlated with a lower white matter integrity of the lesioned lateral corticospinal tract and higher white matter integrity of the contralesional medial reticulospinal tract.

\section{Discussion}

Previous human brain imaging studies of unilateral subcortical stroke have frequently reported the damage to the corticospinal tract and its link with greater motor impairment ${ }^{1-8}$. While there are some other sensorimotor pathways passing through lesioned subcortical regions as well, less is known about other altered sensorimotor tracts post unilateral stroke and their role in motor impairment. An important reason for this lack of knowledge is that main descending and ascending brain pathways mostly overlap in the brain and delineate from each other in the at the side of lesioned hemisphere, we observed decreased white matter integrity in corticospinal and bulbospinal tracts, including medial reticulospinal, lateral reticulospinal, and medial longitudinal fasciculus (i.e., descending medial vestibulospinal tract) in individuals with stroke compared to controls (Fig. 1, tracts in red-yellow color). Consistent with our hypotheses, at the side of 
a
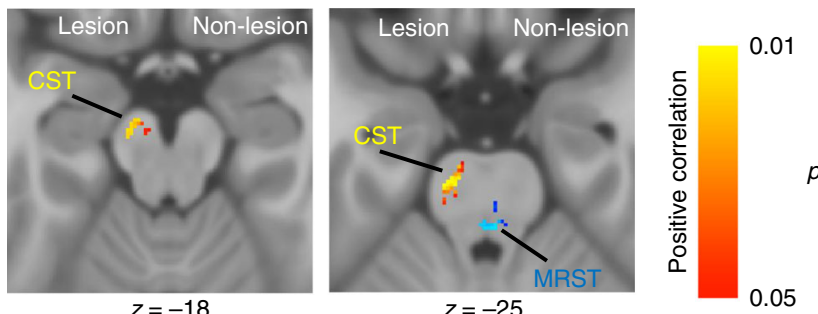

b
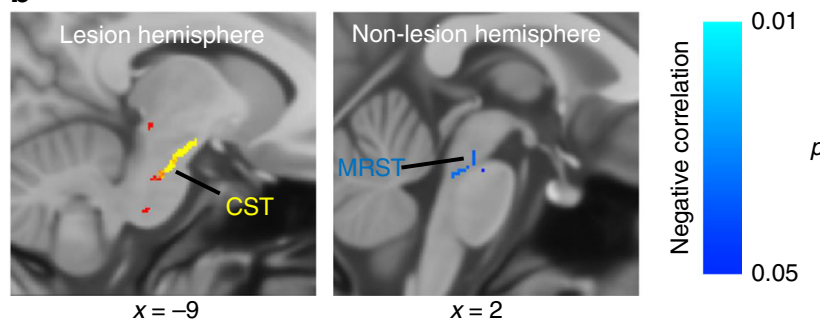

Fig. 2 Tracts with significant correlation between white matter integrity changes and motor impairment. a Axial view of the tract with positive correlation (red-yellow) and negative correlation (blue) between white matter integrity and motor impairment (Fugl-Meyer assessment, FMA) in individuals with stroke. Corticospinal tract (CST) of the lesion hemisphere showed significant positive correlation with impaired motor performance in individuals with stroke. Medial reticulospinal tract (MRST) at the side of non-lesioned hemisphere showed significant negative correlation with impaired motor performance in individuals with stroke. b Sagittal view of the tracts with significant positive (red-yellow) or negative (blue) correlation between white matter integrity and impaired motor performance in individuals with stroke. (Statistical test: within-group voxelwise correlation analysis, using the threshold-free cluster enhancement and $p<0.05)$

brainstem ${ }^{9-12}$, while the previous studies have only investigated the morphological changes in the brain. Additionally, these tracts also continue to pass through separate regions in the spinal $\operatorname{cord}^{9,10}$

In this study, we used high-resolution structural MRI (DTI) of the brainstem and cervical spinal cord and unbiased voxel-wise analyses that, to the best of our knowledge, constitutes the only approach to identify all the sensorimotor pathways with white matter changes after unilateral subcortical stroke. Our results from the brainstem analyses show that the corticospinal and bulbospinal tracts (i.e., medial and lateral reticulospinal tracts and descending medial vestibulospinal tracts) at the side of lesioned hemisphere had decreased white matter integrity in individuals with stroke compared to controls. It is worth noting that these corticospinal and cortico-bulbospinal pathways pass together through the internal capsule-the lesioned region.

Our findings from spinal cord analyses further revealed decreased white matter integrity in cuneate fasciculus, gracile fasciculus, and tectospinal tract of paretic (contralesional) side, which are also linked with the lesioned hemisphere. Decreased white matter integrity of gracile and cuneate fasciculi clearly provides support to previous studies that indicate impairments in tactile and proprioceptive sensations post unilateral stroke $e^{43-46}$.

Last but not least, the results from both brainstem and cervical spinal cord analyses indicated increased white matter integrity of the medial reticulospinal tract, which gets corticobulbar projections from non-lesioned hemisphere motor cortices and projects ipsilaterally from reticular nuclei to spinal $\operatorname{cord}^{9-12}$. The combination of decreased white matter integrity in a number of sensorimotor pathways of the lesioned hemisphere and increased white matter integrity of the medial reticulospinal tract of the non-lesioned hemisphere is the most striking aspect of our results. This may provide an explanation for a large number of previous studies reporting an increase in activation of motor cortices on non-lesioned hemisphere during use of paretic arm in individuals with stroke $\mathrm{e}^{13-15}$. More interestingly, we found that decreases in corticospinal tract integrity at the lesioned hemisphere and increases in medial reticulospinal tract integrity at the non-lesioned hemisphere in stroke individuals were significantly correlated with their motor impairment. These results imply that motor impairments post unilateral stroke are associated with the damage to the corticospinal tract of lesioned hemisphere and also with increased reliance on the medial reticulospinal tract of nonlesion hemisphere. The positive correlation between increased white matter integrity of contralesional medial reticulospinal tract and impairment severity in these individuals is consistent with previous reports of correlation between impairment severity and greater shift of activity to contralesional hemisphere ${ }^{17-23}$. These results have implications for rehabilitation interventions post unilateral stroke. Next, we need to further investigate the role of medial reticulospinal tract in motor control through functional experiments; this knowledge will help us optimize the rehabilitation strategies to work with the innate capabilities of this pathway.

There are a couple of limitations of the current investigation that merit consideration when interpreting the results. First, with regard to the spinal cord MRI spatial resolution, although our acquisition setup comprises the current state of the art in the field of neuroimaging, there is still room for its improvement with the next generation of MRI scanners with better gradient systems. Second, even though we have taken a great care using the most detailed currently known maps of the brainstem and spinal cord tracts from a number of atlases, future improvement in imaging approaches may further inform the correctness of the location of pathways in the human brainstem and spinal cord ${ }^{47}$. Follow-up investigations in the next decades with more advanced MRI scanners and improved knowledge of maps of brainstem and spinal cord tracts may further help refine the anatomical specificity of white matter tracts affected post unilateral stroke.

\section{Methods}

Participants. Thirty-six individuals with chronic hemiparetic unilateral stroke in internal capsule (ischemic or hemorrhagic) and 32 age-matched, gender-matched healthy controls with no neurological or movement disorder were recruited to participate in this study. Stroke subjects were mildly to severely impaired and all sustained a unilateral brain lesion at least 3 years prior to participation in the study Individuals with stroke were recruited through the Northwestern Clinical Neuroscience Research Registry, Ischemic Stroke Registry, and approved flyers. This study was approved by the Institutional Review Board of Northwestern University, and all subjects provided written informed consent prior to participation.

Clinical assessment. For each stroke individual, upper extremity motor assessment $\left(\mathrm{FMA}^{48}\right)$ was performed by a licensed physical therapist. FMA is a performance-based impairment index assessing motor functioning, joint functioning, sensation, and balance in upper limb. Higher values indicate higher motor abilities (less impairment) in these individuals.

Scanning parameters. Brainstem and cervical spinal cord (C2-C5) MRI scans were acquired at Northwestern University Center for Translational Imaging on a 3 T Siemens Prisma scanner with a 64-channel head coil. Brainstem T1-weighted anatomical scans were acquired using an MPRAGE (magnetization-prepared rapid gradient echo) sequence with voxel size $=0.8 \times 0.8 \times 0.8 \mathrm{~mm}$, repetition time $(\mathrm{TR})=9.9 \mathrm{~ms}$, echo time $(\mathrm{TE})=4.6 \mathrm{~ms}$, flip angle $=2^{\circ}$, and field of view $(\mathrm{FOV})=$ $256 \mathrm{~mm}$. Brainstem diffusion-weighted images (DWIs) were collected using spinecho echo-planner imaging with the following parameters: voxel size $=1.5$ isotropic, $\mathrm{TR}=3620 \mathrm{~ms}, \mathrm{TE}=68.4 \mathrm{~ms}$, matrix size $=150 \times 150, \mathrm{FOV}=222 \times 222$ $\mathrm{mm}^{2}$, slice thickness $=1.5 \mathrm{~mm}$, interslice gap $=0 \mathrm{~mm}$, and number of slices covering whole brain and brainstem $=108$. The sequence consisted of diffusion 
a

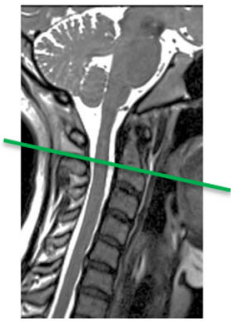

Level C2

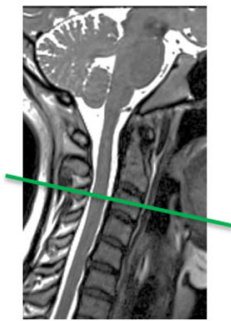

Level C3

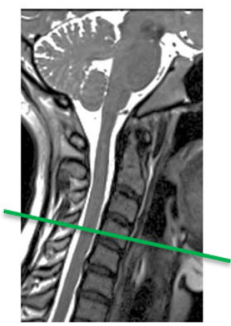

Level C4
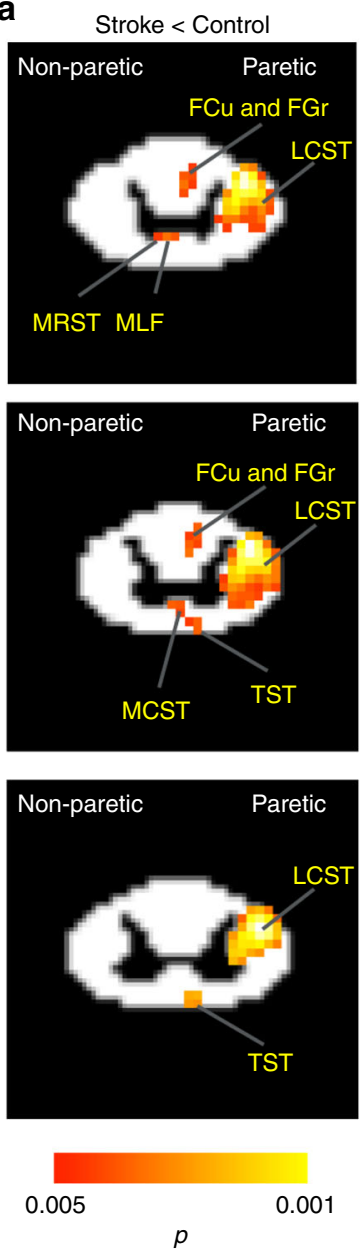

b
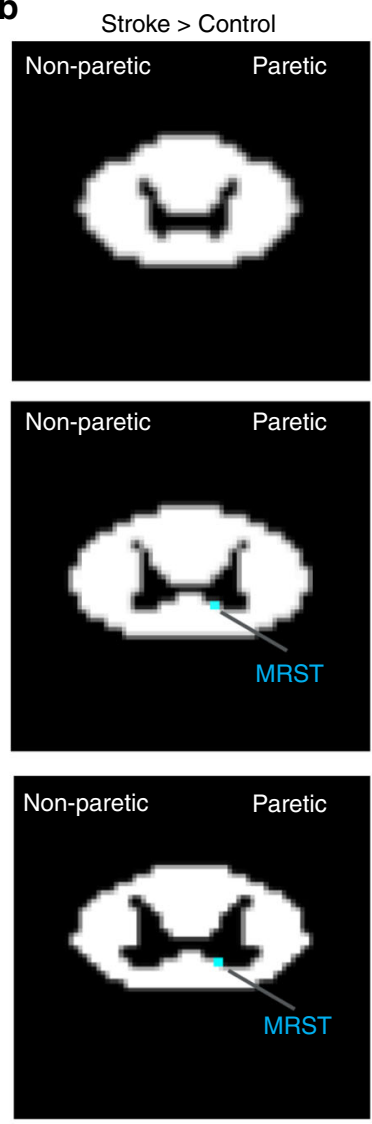

0.005
C

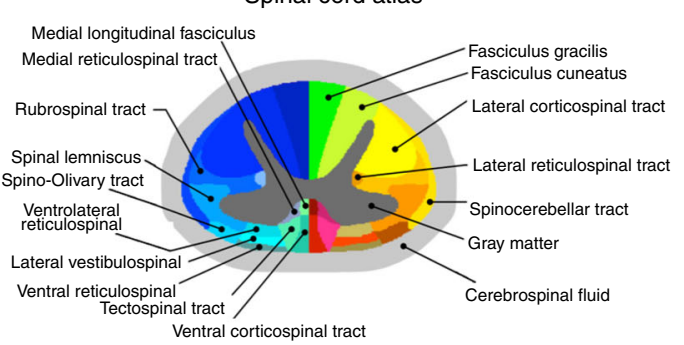

d
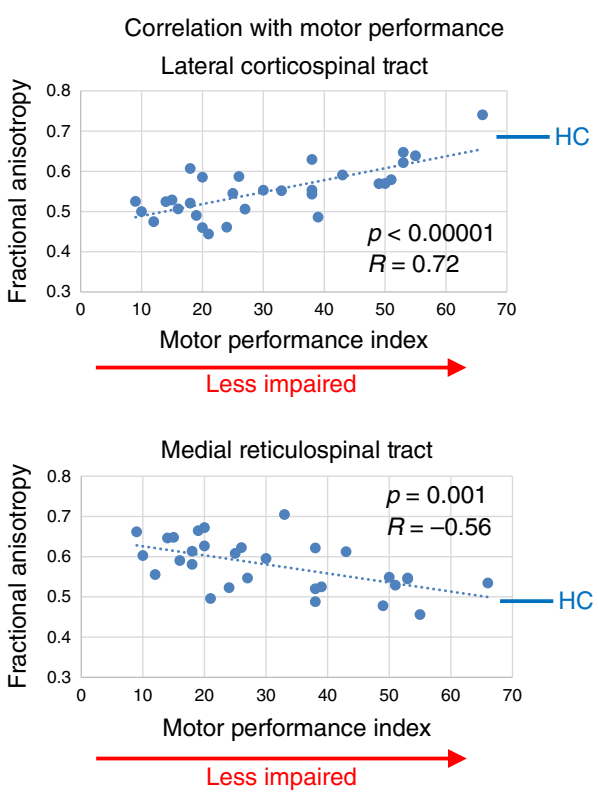

Fig. 3 White matter integrity changes in spinal cord of individuals with stroke compared to controls. a Tracts with significant decrease (red-yellow) in white matter integrity (fractional anisotropy (FA)). When compared to controls, individuals with stroke had significant decrease in white matter integrity of lateral corticospinal tract (LCST), cuneate fasciculus (FCu), gracile fasciculus (FGr), and tectospinal tract (TST) of the paretic side. These individuals also showed decreased white matter integrity in medial reticulospinal tract (MRST), medial longitudinal fasciculus (MLF), and medial corticospinal tract (MCST) of non-paretic side, which project ipsilaterally from the lesioned hemisphere. b Tracts with significant increase (blue) in white matter integrity. Medial reticulospinal tract of paretic side, which projects from non-lesioned motor cortices, had significant increased white matter integrity in individuals with stroke compared to controls. (Statistical test: between-group two-sample $t$ test. Voxel-wise $p<0.005$ was applied and the cluster-level corrected at $p=0.05$.) c High-resolution spinal cord white matter atlas derived from the Gray's Anatomy Atlas, adapted from ref. ${ }^{42}$. $\mathbf{d}$ Correlation of significant white matter integrity changes in individuals with stroke with their motor impairment level. Decreases in lateral corticospinal tract in individuals with stroke had significant positive correlation with their impaired motor performance. Increases in medial reticulospinal tract in individuals with stroke showed a negative correlation with their impaired motor performance. Paretic refers to the side of non-lesioned hemisphere (contralesional). Blue line with abbreviation $\mathrm{HC}$ indicates the mean of FA value in healthy controls for lateral corticospinal tract (equal to 0.68 ) and medial reticulospinal tract (equal to 0.49 ). Fugl-Meyer Assessment (FMA) score is missing for one participant. Source data are provided as a Source Data file

weighting of $1000 \mathrm{~s} / \mathrm{mm}^{2}$ in 60 different directions and 8 scans with no diffusion weighting $\left(b=0 \mathrm{~s} / \mathrm{mm}^{2}\right)$.

Cervical spinal cord T2-weighted anatomical scans were collected with the resolution $=0.8 \times 0.8 \times 0.8, \mathrm{TR}=1500 \mathrm{~ms}$, TE $=100 \mathrm{~ms}$, FOV $=256 \times 256 \mathrm{~mm}^{2}$, number of slices $=64$, and scan duration $=4: 35 \mathrm{~min}$. Cervical spinal cord DWI scans were collected from level C2 to C5 with four acquisitions averaged offline with the following parameters: in-plane resolution $=0.8 \times 0.8, \mathrm{TR}=\sim 600 \mathrm{~ms}$ (depends on heart beat), $\mathrm{TE}=61 \mathrm{~ms}$, slice thickness $=5 \mathrm{~mm}$, interslice gap $=0$ $\mathrm{mm}, \mathrm{FOV}=86 \mathrm{~mm}^{2}$, number of slices $=15, b$ value $=1000$ in 30 directions, 4 images with $b=0$, and scan duration $=6: 35 \mathrm{~min}$. For a better gray matter-white matter segmentation of spinal cord, we also collected a $\mathrm{T} 22^{\star}$-weighted scan that used multi-echo recombined gradient echo with three echoes at 5,10 , and $20 \mathrm{~ms}$, TR = $769 \mathrm{~ms}$, in-plane resolution $=0.4 \times 0.4 \mathrm{~mm}^{2}$, slice thickness $=5 \mathrm{~mm}$, number of slices $=15$, and the scan duration $=5: 11 \mathrm{~min}$.

Brainstem DTI data preprocessing. The DWIs were pre-processed using the FMRIB Software Library version 5.0.11 (FSL: Oxford Centre for Functional MRI of the Brain, UK; http://www.fmrib.ox.ac.uk/fsl//49,50. Raw DTI data were corrected for eddy current distortions using the Eddy Current Correction tool and for motion using the McFlirt Motion Correction tool. The FMRIB Brain Extraction Tool ${ }^{51}$ was then used for skull stripping. Diffusion tensors were fitted at each voxel and FA images were created using the DTIFIT tool of FMRIB FDT toolbox version $5.0^{49}$.

Brainstem tract-based spatial statistics analysis. Majority of stroke participants had lesion in the left hemisphere. For those individuals with lesions in the right hemisphere, FA maps were flipped so that all subjects had lesions in the left hemisphere for group analysis. Voxel-wise statistical analysis of the DTI FA images was carried out using tract-based spatial statistics (TBSS ${ }^{52}$ ) in FSL. First, all subjects' FA images were aligned to the standard $1 \times 1 \times 1 \mathrm{~mm}^{3}$ MNI152 template in FSL, using the non-linear registration tool (FNIRT). Next, the mean FA image was created and thinned to create a mean FA skeleton representing the centers of all tracts, using a threshold of 0.25 . Each subject's aligned FA map was then projected onto this skeleton resulting in each subject's skeletonized FA image, and then masked by brainstem standard template to limit the analysis to the brainstem. 
Group differences in voxel-wise FA were examined by entering each subjects' brainstem skeletonized FA into a general linear model (two-sample $t$ test) design matrix with non-parametric permutation testing, using the Randomize tool in FSL (50,000 permutations). The results were thresholded at $p=0.05$ (corrected), using the threshold-free cluster enhancement option to find clusters without setting an initial cluster level ${ }^{53}$

To further test any association between these group differences and motor impairment in individuals with stroke, we ran a within-group voxel-wise correlation between stroke participants' brainstem skeletonized FA masked by group differences and each subject's clinical assessment of performance. Results were corrected at $p=0.05$ using the threshold-free cluster enhancement option to find the clusters with significant positive or negative correlation with motor impairment level in stroke individuals.

Spinal cord data preprocessing. All spinal cord images were visually inspected and excluded if motion, low signal, or artifacts were present. Data preprocessing was done using spinal cord toolbox version 3.2.2 (SCT; https://www.nitrc.org/ projects/sct $/)^{54}$. Automatic spinal cord segmentation was performed on T2WI, $\mathrm{T} 2^{\star} \mathrm{WI}$, and DWI scans ${ }^{55}$. Images were nonlinearly registered to the MNI-PolyAMU template/atlas in $\mathrm{SCT}^{42}$. T2 ${ }^{*} \mathrm{WI}$ image, which has a strong contrast between gray matter and white matter, was further analyzed with automatic segmentation of gray matter and white matter, and was used to refine the registrations to the template.

DTI data were corrected for motion and registered to the template. FA maps were then created for each subject and registered to the template, using the same refined registration matrix.

Spinal cord DTI voxel-wise statistical analyses. For those individuals with lesions in the right hemisphere (left paretic side), spinal cord FA maps were flipped so that all subjects included in the group analysis had the paretic side presented on right. Statistical analysis of spinal cord DTI data was conducted using SPM12 (Statistical Parametric Mapping; https://www.fil.ion.ucl.ac.uk/spm/software/ spm12/) software on MATLAB 2018a (https:/www.mathworks.com/products/ matlab.html). Statistical analysis was done by entering the individual subject FA maps into between-group two-sample $t$ test analyses. Since some subjects had a lower signal-to-noise ratio at the vertebral level $\mathrm{C} 5$, the between-group analysis was limited to the $\mathrm{C} 2-\mathrm{C} 4$ level. Voxel-wise $p=0.005$ was applied and the cluster level corrected at $p=0.05$.

Reporting summary. Further information on research design is available in the Nature Research Reporting Summary linked to this article.

\section{Data availability}

The data underlying the findings of this study are available from the corresponding author upon reasonable request. The source data underlying Fig. $3 \mathrm{~d}$ are provided as a Source Data file.

Received: 25 February 2019 Accepted: 27 June 2019

Published online: 06 August 2019

\section{References}

1. Werring, D. J. et al. Diffusion tensor imaging can detect and quantify corticospinal tract degeneration after stroke. J. Neurol. Neurosurg. Psychiatry 69, 269-272 (2000).

2. Thomalla, G. et al. Diffusion tensor imaging detects early Wallerian degeneration of the pyramidal tract after ischemic stroke. Neuroimage 22, 1767-1774 (2004).

3. Ward, N. S. et al. Motor system activation after subcortical stroke depends on corticospinal system integrity. Brain 129, 809-819 (2006)

4. Lindenberg, R. et al. Structural integrity of corticospinal motor fibers predicts motor impairment in chronic stroke. Neurology 74, 280-287 (2010).

5. Thomalla, G., Glauche, V., Weiller, C. \& Rother, J. Time course of wallerian degeneration after ischaemic stroke revealed by diffusion tensor imaging. $J$. Neurol. Neurosurg. Psychiatry 76, 266-268 (2005).

6. Moller, M. et al. Dynamic changes in corticospinal tracts after stroke detected by fibretracking. J. Neurol. Neurosurg. Psychiatry 78, 587-592 (2007).

7. Watanabe, T. et al. Three-dimensional anisotropy contrast magnetic resonance axonography to predict the prognosis for motor function in patients suffering from stroke. J. Neurosurg. 94, 955-960 (2001).

8. Puig, J. et al. Wallerian degeneration in the corticospinal tract evaluated by diffusion tensor imaging correlates with motor deficit 30 days after middle cerebral artery ischemic stroke. Am. J. Neuroradiol. 31, 1324-1330 (2010).

9. Gray, H. et al. Gray's Anatomy: The Anatomical Basis of Clinical Practice (Elsevier, Edinburgh, 2016).
10. Haines, D. E. Neuroanatomy: An Atlas of Structures, Sections, and Systems, Vol. 153 (Lippincott Williams \& Wilkins, Philadelphia, 2004).

11. Mai, J. K. \& Paxinos, G. The Human Nervous System (Academic Press, New York, 2011)

12. Vanderah, T. \& Gould, D. J. Nolte's The Human Brain E-Book: An Introduction to its Functional Anatomy (Elsevier Health Sciences, Philadelphia, 2015).

13. Chollet, F. et al. The functional anatomy of motor recovery after stroke in humans: a study with positron emission tomography. Ann. Neurol. 29, 63-71 (1991).

14. Grefkes, C. et al. Cortical connectivity after subcortical stroke assessed with functional magnetic resonance imaging. Ann. Neurol. 63, 236-246 (2008).

15. Weiller, C., Chollet, F., Friston, K. J., Wise, R. J. \& Frackowiak, R. S. Functional reorganization of the brain in recovery from striatocapsular infarction in man. Ann. Neurol. 31, 463-472 (1992).

16. Ward, N. S., Brown, M. M., Thompson, A. J. \& Frackowiak, R. S. Neural correlates of motor recovery after stroke: a longitudinal fMRI study. Brain 126, 2476-2496 (2003).

17. Cao, Y., Olhaberriague, L., Vikingstad, E. M., Levine, S. R. \& Welch, K. M. D. Pilot study of functional MRI to assess cerebral activation of motor function after poststroke hemipareseis. Stroke 29, 112-122 (1998).

18. Carey, J. R. et al. Analysis of fMRI and finger tracking training in subjects with chronic stroke. Brain 125, 773-788 (2002).

19. Cramer, S. C., Finklestein, S. P., Schaechter, J. D., Bush, G. \& Rosen, B. R. Activation of distinct motor cortex regions during ipsilateral and contralateral finger movements. J. Neurophysiol. 81, 383-387 (1999).

20. Cramer, S. C., Moore, C. I., Finklestein, S. P. \& Rosen, B. R. A pilot study of somatotopic mapping after cortical infarct. Stroke 31, 668-671 (2000).

21. Cramer, S. C. et al. A functional MRI study of subjects recovered from hemiparetic stroke. Stroke 28, 2518-2527 (1997).

22. Marshall, R. S. et al. Evolution of cortical activation during recovery from corticospinal tract infarction. Stroke 31, 656-661 (2000).

23. McPherson, J. G. et al. Progressive recruitment of contralesional corticoreticulospinal pathways drives motor impairment post stroke. J. Physiol. 596, 1211-1225 (2018)

24. Baker, S. N., Zaaimi, B., Fisher, K. M., Edgley, S. A. \& Soteropoulos, D. Pathways mediating functional recovery. Prog. Brain Res. 218, 389-412 (2015).

25. Herbert, W. J., Powell, K. \& Buford, J. A. Evidence for a role of the reticulospinal system in recovery of skilled reaching after cortical stroke: initial results from a model of ischemic cortical injury. Exp. Brain Res. 233, 3231-3251 (2015).

26. Zaaimi, B., Soteropoulos, D. S., Fisher, K. M., Riddle, C. N. \& Baker, S. N. Classification of neurons in the primate reticular formation and changes after recovery from pyramidal tract lesion. J. Neurosci. 38, 6190-6206 (2018).

27. Schwerin, S. et al. Ipsilateral versus contralateral cortical motor projections to a shoulder adductor in chronic hemiparetic stroke: implications for the expression of arm synergies. Exp. Brain Res. 185, 509-519 (2008).

28. Dewald, J. P. A., Pope, P. S., Given, J. D., Buchanan, T. S. \& Rymer, W. Z. Abnormal muscle coactivation patterns during isometric torque generation at the elbow and shoulder in hemiparetic subjects. Brain 118, 495-510 (1995).

29. Mukherjee, P., Berman, J. I., Chung, S. W., Hess, C. P. \& Henry, R. G. Diffusion tensor MR imaging and fiber tractography: theoretic underpinnings. Am. J. Neuroradiol. 29, 632-641 (2008).

30. Pierpaoli, C. et al. Water diffusion changes in Wallerian degeneration and their dependence on white matter architecture. Neuroimage 13, 1174-1185 (2001).

31. Virta, A., Barnett, A. L. \& Pierpaoli, C. Visualizing and characterizing white matter fiber structure and architecture in the human pyramidal tract using diffusion tensor MRI. Magn. Reson. Imaging 17, 1121-1133 (1999).

32. Heise, V., Filippini, N., Ebmeier, K. P. \& Mackay, C. E. The APOE varepsilon4 allele modulates brain white matter integrity in healthy adults. Mol. Psychiatry 16, 908-916 (2011).

33. Kraus, M. F. et al. White matter integrity and cognition in chronic traumatic brain injury: a diffusion tensor imaging study. Brain 130, 2508-2519 (2007).

34. Vernooij, M. W. et al. White matter atrophy and lesion formation explain the loss of structural integrity of white matter in aging. Neuroimage 43, 470-477 (2008).

35. Bengtsson, S. L. et al. Extensive piano practicing has regionally specific effects on white matter development. Nat. Neurosci. 8, 1148-1150 (2005).

36. Lövdén, M. et al. Experience-dependent plasticity of white-matter microstructure extends into old age. Neuropsychologia 48, 3878-3883 (2010).

37. Scholz, J., Klein, M. C., Behrens, T. E. \& Johansen-Berg, H. Training induces changes in white-matter architecture. Nat. Neurosci. 12, 1370-1371 (2009). 
38. Cohen-Adad, J. et al. Demyelination and degeneration in the injured human spinal cord detected with diffusion and magnetization transfer MRI. Neuroimage 55, 1024-1033 (2011).

39. Martin, A. R. et al. Translating state-of-the-art spinal cord MRI techniques to clinical use: a systematic review of clinical studies utilizing DTI, MT, MWF, MRS, and fMRI. Neuroimage Clin 10, 192-238 (2016).

40. Renoux, J. et al. MR diffusion tensor imaging and fiber tracking in inflammatory diseases of the spinal cord. Am. J. Neuroradiol. 27, 1947-1951 (2006).

41. Paxinos, G., Xu-Feng, H., Sengul, G. \& Watson, C. in The Human Nervous System 3rd edn (eds Mai, J. L. \& Paxinos, G.) 260-327 (Elsevier, Amsterdam, 2012).

42. Levy, S. et al. White matter atlas of the human spinal cord with estimation of partial volume effect. Neuroimage 119, 262-271 (2015)

43. Dukelow, S. P. et al. Quantitative assessment of limb position sense following stroke. Neurorehabil. Neural Repair 24, 178-187 (2010).

44. Kim, J. S. \& ChoiKwon, S. Discriminative sensory dysfunction after unilateral stroke. Stroke 27, 677-682 (1996).

45. Tyson, S. F., Hanley, M., Chillala, J., Selley, A. B. \& Tallis, R. C. Sensory loss in hospital-admitted people with stroke: characteristics, associated factors, and relationship with function. Neurorehabil. Neural Repair 22, 166-172 (2008).

46. Carey, L. M. Somatosensory loss after stroke. Crit. Rev. Phys. Rehabil. Med. 7, https://doi.org/10.1615/CritRevPhysRehabilMed.v7.i1.40 (1995).

47. Duval, T. et al. Axons morphometry in the human spinal cord. Neuroimage 185, 119-128 (2019).

48. Fugl-Meyer, A. R., Jaasko, L., Leyman, I., Olsson, S. \& Steglind, S. The poststroke hemiplegic patient. 1. A method for evaluation of physical performance. Scand. J. Rehabil. Med. 7, 13-31 (1975).

49. Behrens, T. E. et al. Characterization and propagation of uncertainty in diffusion-weighted MR imaging. Magn. Reson. Med. 50, 1077-1088 (2003).

50. Smith, S. M. et al. Advances in functional and structural MR image analysis and implementation as FSL. Neuroimage 23(Suppl. 1), S208-S219 (2004).

51. Smith, S. M. Fast robust automated brain extraction. Hum. Brain Mapp. 17, 143-155 (2002).

52. Smith, S. M. et al. Tract-based spatial statistics: voxelwise analysis of multisubject diffusion data. Neuroimage 31, 1487-1505 (2006).

53. Smith, S. M. \& Nichols, T. E. Threshold-free cluster enhancement: addressing problems of smoothing, threshold dependence and localisation in cluster inference. Neuroimage 44, 83-98 (2009).

54. De Leener, B. et al. SCT: Spinal Cord Toolbox, an open-source software for processing spinal cord MRI data. Neuroimage 145, 24-43 (2017).

55. De Leener, B., Kadoury, S. \& Cohen-Adad, J. Robust, accurate and fast automatic segmentation of the spinal cord. Neuroimage 98, 528-536 (2014).

\section{Acknowledgements}

We are indebted to the individuals with stroke who participated in this study. We thank Azmi Banibaker, ARRT, for his assistance with MRI data collection, and Kevin Wilkins for his insightful comments on statistical analyses. This research was supported by funding from the NIH R01HD039343 and R01NS105759 awarded to J.P.A.D. and NIH NRSA F31NS105486 awarded to H.K.

\section{Author contributions}

H.K. and J.P.A.D. designed the study. H.K. and J.C.-A. set up the spinal cord imaging protocol and analysis pipeline. H.K. collected the data and analyzed the brainstem and spinal cord data. H.K., J.P.A.D. and J.C.-A. wrote the manuscript.

\section{Additional information}

Supplementary Information accompanies this paper at https://doi.org/10.1038/s41467 019-11244-3.

Competing interests: The authors declare no competing interests.

Reprints and permission information is available online at http://npg.nature.com/ reprintsandpermissions/

Peer review information: Nature Communications thanks Hanna Braass, Cornelius Weiller and other anonymous reviewer(s) for their contribution to the peer review of this work.

Publisher's note: Springer Nature remains neutral with regard to jurisdictional claims in published maps and institutional affiliations.

cc (i) Open Access This article is licensed under a Creative Commons Attribution 4.0 International License, which permits use, sharing, adaptation, distribution and reproduction in any medium or format, as long as you give appropriate credit to the original author(s) and the source, provide a link to the Creative Commons license, and indicate if changes were made. The images or other third party material in this article are included in the article's Creative Commons license, unless indicated otherwise in a credit line to the material. If material is not included in the article's Creative Commons license and your intended use is not permitted by statutory regulation or exceeds the permitted use, you will need to obtain permission directly from the copyright holder. To view a copy of this license, visit http://creativecommons.org/licenses/by/4.0/.

(C) The Author(s) 2019 